\title{
Nuclear receptor co-regulator Krüppel-like factor 9 and prohibitin 2 expression in estrogen-induced epithelial cell proliferation in the mouse uterus
}

\author{
J M P Pabona*, M C Velarde*,+, Z Zeng, F A Simmen and R C M Simmen \\ Department of Physiology and Biophysics, University of Arkansas for Medical Sciences and Arkansas Children's Nutrition Center, 1212 Marshall Street, Little \\ Rock, Arkansas 72202, USA \\ (Correspondence should be addressed to R C M Simmen; Email: simmenrosalia@uams.edu) \\ *(J M P Pabona and M C Velarde contributed equally to this study and are considered as co-first authors) \\ ${ }^{+} \mathrm{M} C$ Velarde is now at Department of OB/GYN and Reproductive Sciences, University of California San Francisco, San Francisco, California 94143, USA
}

\begin{abstract}
Estrogen, acting through its cognate receptor estrogen receptor$\alpha($ ESR 1$)$, is a critical regulator of uterine endometrial epithelial proliferation. Although the dynamic communication between endometrial stromal (ST) and epithelial cells is considered to be an important component in this process, key molecular players in particular compartments remain poorly defined. Here, we used mice null for Krüppel-like factor 9 (KLF9) to evaluate the contribution of this nuclear protein in ST-epithelial interactions underlying proliferative effects of estrogen. We found that in ovariectomized mice administered estradiol-17 $\beta\left(\mathrm{E}_{2}\right)$ for $24 \mathrm{~h}$, Klf9 null mutation resulted in lack of $\mathrm{E}_{2}$-induced proliferative response in all endometrial compartments. We demonstrated a negative association between Klf9 expression and nuclear levels of ESR1 transcriptional corepressor prohibitin (PHB) 2 in uterine ST and epithelial cells of $\mathrm{E}_{2}$-treated wild-type (WT) and
\end{abstract}

Klf9 null mice. In early pregnancy uteri of WT mice, the temporal pattern of Klf 9 transcript levels was inversely associated with that of Phb2. Deletion of Klf9 up-regulated uterine Phb2 expression and increased PHB2 nuclear localization in endometrial ST and epithelial cells, with no effects on the expression of the related Phb1. In the human endometrial ST cell line treated with $\mathrm{E}_{2}$ for $24 \mathrm{~h}$, Klf9 siRNA targeting augmented PHB2 transcript and increased nuclear PHB2 protein levels, albeit this effect was not to the extent seen in vivo with Klf9 null mutants. Our findings suggest a novel mechanism for control of estrogen-induced luminal epithelial proliferation involving ST KLF9 regulation of paracrine factor(s) to repress epithelial expression of corepressor PHB2.

Journal of Endocrinology (2009) 200, 63-73

\section{Introduction}

Estrogen (E) control of cell proliferation is a complex process that is subject to regulation at many levels. The nuclear receptor/transcription factor estrogen receptor- $\alpha$ (ESR1) is the key regulatory participant, transducing $\mathrm{E}$ action by binding the ligand to form a complex that, upon homodimerization, interacts with various co-regulators to target E-responsive gene promoters, leading to transcriptional activation, and the synthesis of gene products that modify cellular phenotype and behavior (Tsai \& O’Malley 1994, Hall et al. 2001). Nuclear receptor co-regulator proteins, acting as coactivators or corepressors contribute to ESR1 transactivity and exert their effects by direct or indirect interactions with ESR1 (McKenna \& O'Malley 2002, Lonard \& O'Malley 2006, Green \& Carroll 2007). Examples of co-factors that directly interact with ligandbound ESR 1 at its activation function domains include the forkhead protein FOXA1 and the p160 protein family members NCOA7/ERA160/NCOA1, NCOA2, and NCOA3 (Yahata et al. 2001, Hu et al. 2002, Dutertre \& Smith
2003, Wang et al. 2007). Co-regulators that influence ESR1 transactivation of target genes indirectly alter chromatin structure by facilitating histone acetylation/deacetylation and methylation/demethylation, and by post-translational modifications of other co-regulators and transcription factors within the transcriptional complex (Stenoien et al. 2001, McKenna \& O'Malley 2002, Green \& Carroll 2007). The reports on the formation of an extranuclear complex between ligand-bound ESR1 and NCOA3 (Zheng et al. 2005) and the increased transcription of ESR 1 target genes upon MAPK phosphorylation of NCOA3 (Font de Mora \& Brown 2000) provide evidence for the increasingly complex and less predictable mechanisms underlying co-regulator participation in ESR1 signaling.

We previously reported that the nuclear protein Krüppel-like factor 9 (KLF9), previously designated basic transcription element-binding protein-1 (Imataka et al. 1992) and a member of the Sp family of transcription factors (Suske et al. 2005) may function as a co-regulator of steroid hormone receptor signaling in the uterine endometrium (Zhang et al. 2002, 2003). We found 
that KLF9 exerts its effects on progesterone receptor (PGR) and ESR1 actions by distinct mechanisms. KLF9 promotes PGR transactivation through functional and physical interactions with PGR-B and to a lesser extent, PGR-A at progesterone (P)responsive gene promoters (Zhang et al. 2003, Velarde et al. 2006). By contrast, KLF9 can inhibit ESR 1 transactivity in vitro in a high-estrogen environment by promoting estradioldependent down-regulation of ESR1 expression through enhancement of the association of ESR1 to GC-rich motifs within its promoter (Velarde et al. 2007). Based on these studies, we concluded that KLF9 may influence both PGR and ESR1 genomic pathways to favor $\mathrm{P}$-induced cell differentiation (Velarde et al. 2007). Nonetheless, while the latter presented an attractive model integrating KLF9 in the opposing actions of $\mathrm{E}$ and $\mathrm{P}$ in the uterus, it did not account for our earlier findings that in the endogenous E-dominated environment of early pregnancy, uterine endometrial cells of Klf9 null mice exhibited delayed (by $24 \mathrm{~h}$ ) and attenuated proliferation relative to wildtype (WT) counterparts (Velarde et al. 2005). The decreased numbers of implanting embryos in Klf9 null mutants suggested that the altered pattern of proliferation of endometrial cells with Klf9 ablation resulted in developmental asynchrony between the uterine luminal epithelium (LE) and the implantation-ready embryo, leading to subfertility (Simmen et al. 2004, Velarde et al. 2005). Thus, KLF9 may function as a positive regulator of ESR1 signaling to influence cell proliferation, with important consequences on pregnancy outcome.

Prohibitin (PHB) 2, also designated as repressor of estrogen receptor activity (REA), is a $37 \mathrm{kDa}$ protein exhibiting high homology to the putative tumor suppressor protein PHB1 (Montano et al. 1999). PHB1 and PHB2 are highly conserved proteins in eukaryotic cells, with similar functions as inhibitors of cellular proliferation (Mishra et al. 2006). Unlike PHB1, however, which represses signaling of numerous steroid hormone receptors (Wang et al. 2004, Mussi et al. 2006, Gamble et al. 2007, He et al. 2007), PHB2 demonstrates selective repression of ER activity (Montano et al. 1999), possibly in a cell-specific context (Wang et al. 2004), and cooperates with the chicken ovalbumin upstream promoter binding transcription factors I and II to decrease transcription (Kurtev et al. 2004). PHB2 inhibits ESR1 transcriptional activity by competing with p160 co-regulators such as NCOA1 and NCOA3 for binding to ESR1 in the presence of E (Montano et al. 1999, Delage-Morroux et al. 2000, Wang et al. 2004), and by recruiting class I and class II histone deacetylases (Kurtev et al. 2004). Whereas genetic deletion of both $\mathrm{Phb2}$ alleles resulted in embryonic lethality, heterozygous mice displayed phenotypes that are characteristic of overactivated ESR 1 signaling including uterine epithelial hyperplasia coincident with higher expression levels of E-responsive genes, enhanced mammary gland morphogenesis, and delayed mammary gland involution (Park et al. 2005, Mussi et al. 2006). These collective results indicate that $\mathrm{PHB} 2$ functions as an important mediator of $\mathrm{E}$ action in vivo.

In the present study, we show that endometrial epithelial cells of ovariectomized (Ovx) mice with Klf9 null mutation were unresponsive to $\mathrm{E}_{2}$-induced proliferation. We hypothesized that stromal (ST) KLF9 may mediate $\mathrm{E}_{2}$ effects on uterine epithelial cell proliferation by influencing the expression of specific ESR1 co-regulators. We demonstrate that the KLF9mediated increase in proliferation with $\mathrm{E}_{2}$ was negatively associated with uterine $P h b 2$, but not $P h b 1$, expression and with nuclear localization of PHB2 in all endometrial compartments. We further show that this negative linkage between KLF9 and nuclear PHB2 also occurs during the physiological condition of early pregnancy and in an $\mathrm{E}_{2}$-treated human endometrial $\mathrm{ST}$ cell line (HESC). Our results suggest that the repression of epithelial PHB2 expression involving ST KLF9 signaling may be a necessary component in the paracrine regulation of uterine epithelial proliferation by estrogen.

\section{Materials and Methods}

\section{Animals and treatments}

Experiments were carried out in accordance with the protocols approved by the Institutional Animal Care and Use Committee of the University of Arkansas for Medical Sciences. WT and Klf9 mutant (KO) mice lines were propagated and genotyped as described previously (Morita et al. 2003, Simmen et al. 2004). For steroid hormone treatment studies, $\sim 8$-week-old WT and $\mathrm{KO}$ female littermates were subjected to bilateral ovariectomy. Two weeks later, mice were treated with vehicle (sesame oil; $0 \cdot 25 \mathrm{ml}$ ) or $17 \beta$-estradiol $\left(\mathrm{E}_{2}, 125 \mathrm{ng}\right.$ in $0.25 \mathrm{ml}$ sesame oil), and uteri were collected after $24 \mathrm{~h}$. Uterine tissues from WT and $\mathrm{KO}$ mice at day post-coitum (dpc) $2 \cdot 5,3 \cdot 5$, and $4 \cdot 5$ were isolated as described previously (Velarde et al. 2005). The presence of vaginal plug was considered $0.5 \mathrm{dpc}$.

\section{Immunohistochemistry}

Paraffin-embedded uteri from Ovx WT and KO females ( $n=3-5$ mice per genotype per treatment) were serially sectioned, dewaxed in xylene, and rehydrated through a graded alcohol series. Tissue sections were treated with 3\% $\mathrm{H}_{2} \mathrm{O}_{2}$ to quench endogenous peroxidase activity. Antigen unmasking was performed by boiling the sections in Citra Plus (Biogenex, San Ramon, CA, USA) in a microwave oven for $105 \mathrm{~s}$ at power 10 and then for $10 \mathrm{~min}$ at power 1 . After blocking with horse IgG (Vectastain Elite ABC kit, Vector Laboratories, Inc., Burlingame, CA, USA) for $30 \mathrm{~min}$, sections were incubated with the following antibodies to detect expression of proteins: a) mouse monoclonal antibody to proliferating cell nuclear antigen (PCNA) (PC-10, Dako, Carpinteria, CA, USA) at 1:500 dilution; b) rabbit polyclonal antibody to ESR1 (MC-20, Santa Cruz Biotechnology, Inc.) at 1:500 dilution; and c) rabbit polyclonal antibody to $\mathrm{PHB} 2$ (Bethyl Laboratories Inc., Montgomery, TX, USA) at 1:250 dilution. Incubations with anti-PCNA and ESR 1 were carried out for $1 \mathrm{~h}$ at room temperature, whereas incubation with anti-PHB2 antibody was performed overnight at room 
temperature. Following incubation with secondary antibody (horse anti-mouse IgG at 1:1600 dilution or goat anti-rabbit IgG at 1:2000 dilution) for $30 \mathrm{~min}$, sections were stained with 3, $3^{\prime}$-diaminobenzidine tetra-hydrochloride (Dako) and counterstained with hematoxylin. Control sections were processed similarly with the omission of the primary antibody. A total of 1000 ST, 300 luminal epithelial, and 200 glandular epithelial cells were counted on average from at least three randomly selected fields $(200 \times$ magnification) per slide; one to two slides/mouse with $n=3-5$ mice per treatment group per genotype, were evaluated. Results are expressed as \% nuclear-immunopositive cells ((number of nuclear positively staining cells/number of total cells counted) $\times 100$ ).

\section{$R N A$ isolation and quantitative RT-PCR}

Total cellular RNA was prepared from whole uteri or cells by TRIzol reagent (Invitrogen). Integrity of isolated RNAs was confirmed using the RNA 6000 NanoLabChip kit (Agilent Biotechnologies, Palo Alto, CA, USA). RNA samples were reverse transcribed using random primers and a cDNA synthesis kit (Applied Biosystems, Foster City, CA, USA). SYBR Green quantitative RT-PCR was performed as described previously (Velarde et al. 2006). Primer sets were designed to flank an intron to prevent amplification of genomic DNA, using PrimerExpress (Applied Biosystems). Synthetic oligonucleotides were obtained from Integrated DNA Technologies, Inc. (Coralville, IA, USA). The sense and antisense primers for the mouse genes, and the resultant PCR product sizes (in parentheses) were: Klf9, $5^{\prime}$-CGT TGC CCA CTG TGT GAG AA- $3^{\prime}$ and $5^{\prime}$-TTG ATC ATG CTG GGA TGG AA-3' (92 bp); Phb2/Rea, 5'-AGC AGG AAC AGC AC AGA AGA- $3^{\prime}$ and $5^{\prime}$-CGG AGC TTG ATA TAG CCA GGA T-3' (103 bp); and Phb1, 5'-TCC CTT GGG TAC AGA AAC CAA TTA- $3^{\prime}$ and $5^{\prime}$-TGTGAT ATT GAC GTT CTG CAA GTC T-3' (101 bp). For each sample, gene expression was normalized to that of cyclophilin A (Ppia) (sense: $5^{\prime}$-AGA TGC CAG GAC CTG TAT GCT T-3'; antisense: $5^{\prime}$-TGT GCC AGG GTG GTG ACT TTA-3 ${ }^{\prime}$ ) as internal reference. Human primers (sense and antisense respectively) for KLF9, $P H B 2 / R E A$, and the internal reference gene RPL7 were as follows: KLF9, $5^{\prime}$-TGG CTG TGG GAA AGT CTA TGG- $3^{\prime}$ and $5^{\prime}$-CTC GTC TGA GCG GGA GAAA CT-3' (124 bp); PHB2/REA, 5'-GAA CAG CGG CAG AAA ATT GTG-3' and $5^{\prime}$-CGA ATC TTG CGA AGT TTG ATG T-3' (105 bp), and RPL7, $5^{\prime}-$ TGCTGT GCC AGA AAC CCT TAA- $3^{\prime}$ and $5^{\prime}$-GCT TCC TCC TTG CCT TTC G-3' (110 bp).

\section{Cell culture, transfection with siRNAs, immunofluorescence, and western blots}

The HESC was a generous gift from Dr Graziella Krikun (Yale University, New Haven, CT, USA). The cell line was maintained in phenol red-free DMEM and Ham's F12 (1:1 vol/vol)medium supplemented with $10 \%$ charcoal-stripped calf serum and $1 \%$ antibiotic/antimycotic solution in an atmosphere of 5\% $\mathrm{CO}_{2}$, as described previously (Krikun et al. 2004). Transfection with Klf9 or scrambled (non-specific) siRNAs (Dharmacon, Waltham, MA, USA) at a final concentration of $50 \mathrm{nM}$ was performed using Lipofectamine 2000 according to the manufacturer's protocol when cells were $\sim 60 \%$ confluent. After $6 \mathrm{~h}$, the transfection mix was replaced with the medium containing $2 \%$ charcoal-stripped calf serum. Cells were incubated for an additional $24 \mathrm{~h}$ in $2 \%$ charcoal-stripped calf serum with added $\mathrm{E}_{2}(10 \mathrm{nM})$ and collected for RNA analyses. Immunofluorescence was performed essentially as described previously (Velarde et al. 2007). In brief, cells were seeded on sterile $22 \mathrm{~mm}$ glass cover slides at a density of $1 \cdot 8 \times 10^{5}$ cells per well and grown overnight. After transfection with siRNAs, cells were incubated in the medium containing $\mathrm{E}_{2}(10 \mathrm{nM})$ in DMSO for $24 \mathrm{~h}$. Cells were fixed in $4 \%$ paraformaldehyde for $10 \mathrm{~min}$ and permeabilized in $0 \cdot 1 \%$ Triton-PBS for $45 \mathrm{~min}$, with $1 \times$ PBS washes after each step. Cells were sequentially incubated in goat serum blocking solution (Vectastain Elite ABC kit) for 30 min and with anti-PHB2 polyclonal antibody (1:250 dilution) overnight. After incubation with biotinylated secondary antibody (Vectastain Elite ABC kit), fluorescence signals were developed, visualized, and quantified for percentage of nuclear staining cells (relative to total number of cells; $\sim 250$ per treatment group; Velarde et al. 2007). Western immunoblots were performed (Velarde et al. 2007) using antirat KLF9 antibody generated in-house (Zhang et al. 2002) and anti- $\alpha$-actinin antibody (Santa Cruz Biotechnology) for loading control.

\section{Data analysis}

Values are presented as mean \pm s.E.M. Data were analyzed using SigmaStat (SPSS Science, Chicago, IL, USA) and evaluated for differences between groups by Student's $t$-test or two-way ANOVA, followed by Tukey's test. $P \leq 0 \cdot 05$ were considered statistically significant.

\section{Results}

Uterine endometrial cells are unresponsive to $E_{2}$-induced proliferation with Klf9 null mutation

To evaluate the proliferative response of uterine endometrial cells to $\mathrm{E}$ as a function of KLF9 expression status, agematched Ovx WT and Klf9 KO littermates were subcutaneously administered $E_{2}$ in sesame oil or sesame oil alone (control), and corresponding uteri were isolated after $24 \mathrm{~h}$. Tissue sections from WT and $\mathrm{KO}$ mice were immunostained for the proliferation marker PCNA (Fig. 1A), and the numbers of immunopositive cells in each endometrial compartment were counted. In WT mice, $\mathrm{E}_{2}$ administration increased PCNA expression in LE, glandular epithelium (GE), and ST cells by at least fourfold over those of corresponding control cells (Fig. 1B). Basal and $\mathrm{E}_{2}$-induced 
A

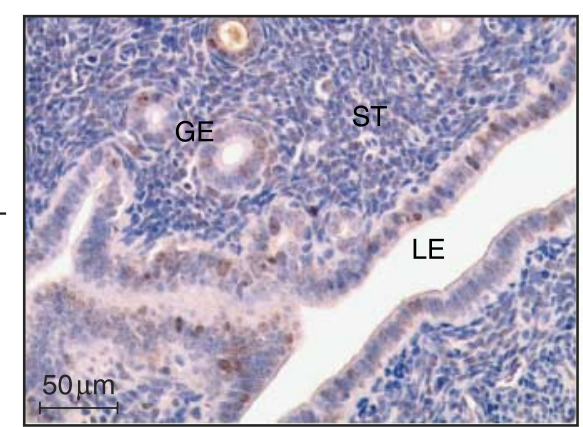

$\mathrm{KO}$

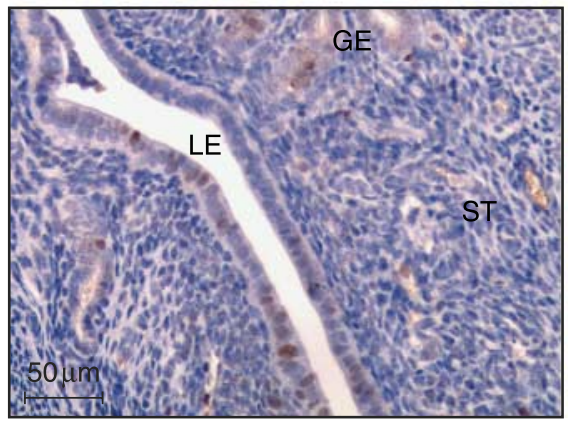

E
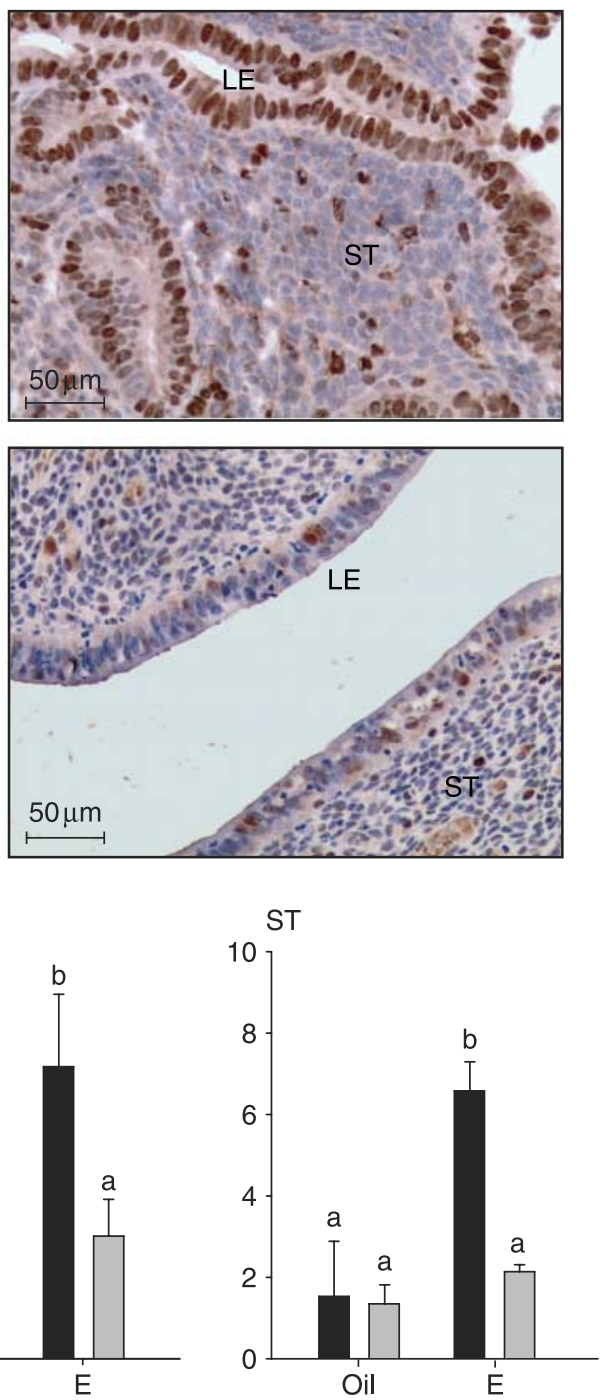

B
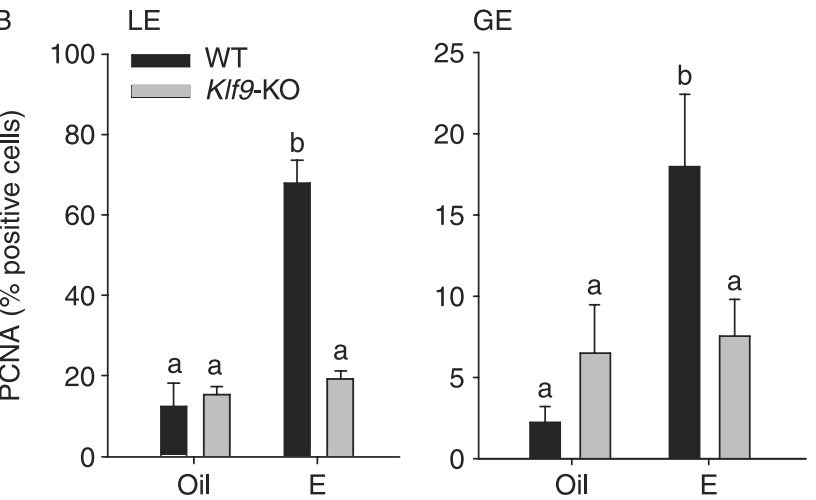

Figure 1 Nuclear PCNA levels in uterine endometrial cells of oil (vehicle) and $\mathrm{E}_{2}$-treated ovariectomized WT and Klf9 null mice. (A) Representative PCNA immunostaining of glandular epithelium (GE), luminal epithelium (LE), and stromal (ST) compartments are shown at $200 \times$ magnification. (B) The percentages of nuclear staining cells are presented as mean \pm S.E.M. $(n=3-5$ mice per treatment per genotype). Significant differences were identified by two-way ANOVA, followed by Tukey's test. Means with different superscripts differed at $P<0 \cdot 05$.

PCNA expression was significantly greater for LE than for GE and ST. By contrast, PCNA immunoreactivity in all endometrial compartments of $\mathrm{E}_{2}$-treated Klf9 null mice did not rise above those of corresponding control cells.

Since $\mathrm{E}_{2}$ predominantly signals through ESR 1 to exert its proliferative effects in the uterus (Dupont et al. 2000), the nuclear levels of this protein in endometrial compartments were evaluated by immunohistochemistry as a function of KLF9 status. Ovx oil-treated WT mice showed robust ESR1 expression in the uterine endometrium. $\mathrm{E}_{2}$ treatment had no effect on nuclear levels of this protein for all cell types, in the presence or absence of KLF9. The percentages of cells positive for nuclear ESR1 in LE from control $(15 \cdot 10 \pm 7 \cdot 43 \%)$ and $E_{2^{-}}$ treated $(22 \cdot 91 \pm 5 \cdot 91 \%)$ WT mice did not differ from those of LE in corresponding control $(10 \cdot 67 \pm 2 \cdot 25 \%)$ and $E_{2}$-treated Klf9 null mice $(11 \cdot 86 \pm 2 \cdot 34 \%)$. Similarly, ST cells from WT (control: $45 \cdot 02 \pm 10 \cdot 38 \%$; $E_{2}$-treated: $38 \cdot 51 \pm 6 \cdot 19 \%$ ) and Klf9 null (control: $35 \cdot 86 \pm 11 \cdot 59 \%$; $E_{2}$-treated: $37 \cdot 78 \pm$ 6.27\%) mice and GE cells from WT (control: 29.21 11.42\%; $E_{2}$-treated: 33.04 $\pm 3 \cdot 23 \%$ ) and Klf9 null (control: $21 \cdot 44 \pm 6 \cdot 65 \% ; E_{2}$-treated: $\left.36 \cdot 23 \pm 13 \cdot 56 \%\right)$ mice showed no differences in percentages of cells positive for nuclear ESR1. 
KLF9 is negatively associated with uterine PHB2 expression and nuclear localization

To understand the mechanism by which ablation of Klf9 expression (predominantly in the ST; Simmen et al. 2004) resulted in the lack of $\mathrm{E}_{2}$-mediated proliferative response in all endometrial compartments, we examined the possibility that KLF9 negatively regulates the expression of the selective ESR1 transcriptional corepressor PHB2 (Montano et al. 1999). Uterine tissues from control (oil) and $\mathrm{E}_{2}$-treated Ovx WT and KO mice were quantified for Phb2 mRNA abundance by qPCR. Control (oil-treated) WT and KO mice showed comparable levels of Phb2 transcripts (Fig. 2A). $\mathrm{E}_{2}$ administration appeared to decrease $(P=0 \cdot 10) \quad P h b 2$ expression in WT mice, relative to corresponding control, and this trend was reversed with loss of Klf9 (Fig. 2A). The expression of the related gene Phb1 was not affected by $\mathrm{E}_{2}$ or by KLF9 expression status (Fig. 2B). Similarly, $\mathrm{E}_{2}$ had no effect on Klf9 transcript levels (Fig. 2C).

We evaluated the presence of $\mathrm{PHB} 2$ protein in uterine tissue sections by immunohistochemistry to determine its spatial distribution pattern, as a function of $\mathrm{E}_{2}$ treatment and KLF9 expression. Using a previously characterized anti-PHB2 antibody (Montano et al. 1999), we found immunoreactive PHB2 in both cytoplasmic and nuclear compartments of GE, LE, and ST cells (Fig. 3A), consistent with the reported localization of this protein in the mitochondrion and nucleus (Fusaro et al. 2003, Mishra et al. 2006). Since E-dependent interactions between PHB2 and ESR 1 occur predominantly in the nucleus to inhibit ESR1 transactivity, nuclear PHB2 expression in endometrial GE, LE, and ST cells was determined in control and $\mathrm{E}_{2}$-treated mice. In oil-treated Ovx WT and $\mathrm{KO}$ mouse uteri, the amount of nuclear PHB2 (measured as \%PHB2-immunopositive cells) were significantly greater in LE $(\sim 20 \%)$ than in GE $(10 \%)$ and ST $(1 \%)$ cells (Fig. 3B). With $\mathrm{E}_{2}$ treatment, the levels of nuclear PHB2 in GE and ST cells of WT uteri did not differ from those of oil-treated controls whereas in LE cells, nuclear PHB2 levels showed a trend to decrease (Fig. 3B), following that seen for corresponding mRNA in whole uteri (Fig. 2A). Loss of Klf9 reversed the possible inhibitory effect of $\mathrm{E}_{2}$ on nuclear PHB2 expression in LE cells to the level of control (non- $\mathrm{E}_{2}$ treated) cells, and increased the percent of nuclear PHB2-immunopositive GE and ST cells, with the latter demonstrating a most dramatic ( 40-fold) enhancement (Fig. 3B).

Uterine PHB2 and KLF9 expression are inversely associated in early pregnancy

We have previously shown that during the E-dominated condition of early pregnancy at dpc $2 \cdot 5$, uterine endometrial cells of Klf9 null mice had negligible BrdU labeling relative to those of WT mice; however, KO cells displayed peak BrdU labeling 1 day later at dpc 3.5, similar to the level of WT cells at dpc $2 \cdot 5$ (Velarde et al. 2005). To determine whether the delayed proliferative response to $\mathrm{E}_{2}$ in $K l f 9$ null uteri was associated with
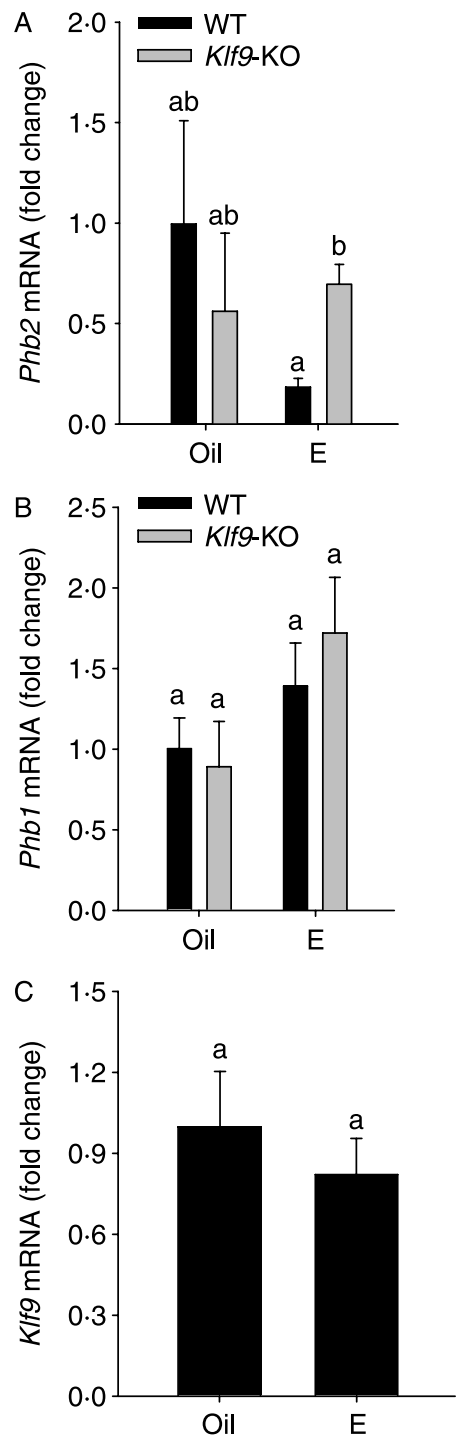

Figure 2 Transcript levels of (A) Phb2, (B) Phb1, and (C) Klf9 in uteri of oil (vehicle) and $E_{2}$-treated ovariectomized WT and/or KIf9 null mice. mRNA expression was quantified by qPCR and normalized to that of the control gene Ppia. Transcript levels (mean \pm S.E.M.) are expressed as fold change relative to WT oil treatment group $(n=3-5$ mice per treatment per genotype). Means with different superscripts differed at $P<0 \cdot 05$.

a transient increase in Phb2 expression, the same WT and KO uterine tissues previously analyzed for proliferation status (Velarde et al. 2005) were evaluated for Phb2 mRNA abundance. In WT mice, $P h b 2$ expression was low at dpc $2 \cdot 5$, significantly increased at dpc 3.5, and remained at that level at dpc $4 \cdot 5$ (Fig. 4A). By contrast, Phb1 mRNA abundance in WT mice (relative to WT dpc 2.5) progressively decreased at early pregnancy (Fig. 4B). The large variations in Phb1 abundance reflect its very low levels of expression, relative to $P h b 2$. The pattern of $P h b 2 \mathrm{mRNA}$ expression was inversely associated with 

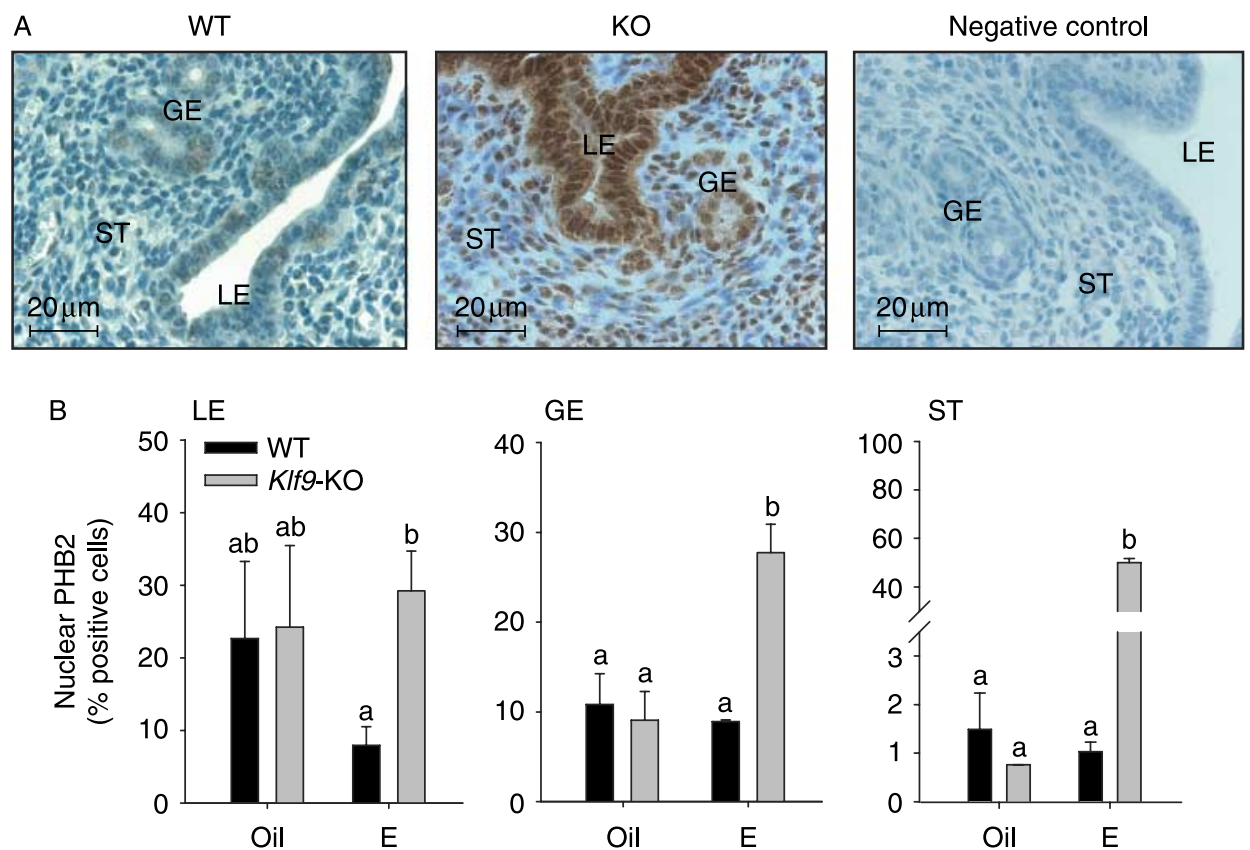

Figure 3 PHB2 levels in uterine endometrial cells of oil (vehicle) and $E_{2}$-treated ovariectomized WT and Klf9 null mice. (A) Representative PHB2 immunostaining of GE, LE, and ST cells of $\mathrm{E}_{2}$-treated WT and Klf9 null $(\mathrm{KO})$ mice are shown at $400 \times$ magnification to demonstrate nuclear localization of immunoreactive PHB2. Negative control was tissue from $E_{2}$-treated WT mice processed similarly except for omission of primary antibody. (B) The percentages of PHB2 nuclear staining cells are presented as mean \pm S.E.M. ( $n=3-5$ mice per treatment per genotype). Significant differences were identified by two-way ANOVA, followed by Tukey's test. Means with different superscripts differed at $P<0 \cdot 05$.

that of Klf9 (Fig. 4C), the latter concordant with the peak of proliferation of luminal epithelial cells at dpc $2 \cdot 5$ and subsequently decreasing at dpc 3.5 and 4.5 (Velarde et al. 2005). In KO mice, Phb2 gene expression was significantly higher than for WT mice at dpc $2 \cdot 5$ and $4 \cdot 5$, while at $\mathrm{dpc} 3 \cdot 5$, no difference in Phb2 expression levels was noted between genotypes (Fig. 4A). Phb1 expression was not affected by the loss of Klf9 expression (Fig. 4B).

Next, immunoreactive PHB2 was localized in uteri of WT and $\mathrm{KO}$ mice at dpc $2 \cdot 5,3 \cdot 5$, and $4 \cdot 5$. Representative immunostained uterine sections from WT and $\mathrm{KO}$ mice at dpc 2.5 are shown (Fig. 5A). In WT mice, immunoreactive PHB2 was present in all endometrial cell types and in the myometrium (not shown), and was localized to both cytoplasmic and nuclear compartments (Fig. 5B). The percentage of nuclear PHB2-positive cells in WT mice was higher in GE and ST cells at dpc 3.5 and 4.5 than at dpc 2.5 (Fig. 5C), following the temporal pattern observed for corresponding mRNA in whole uteri; by contrast, the percentage of nuclear PHB2-positive LE cells did not change across these pregnancy days (Fig. 4A). At dpc $2 \cdot 5$, there was a greater level (by two- to threefold) in the percentage of nuclear PHB2-immunostaining cells for all endometrial cell types of Klf9 null mice, relative to WT counterparts (Fig. 5C). At dpc 3.5, LE and ST, but not GE cells showed similar significant increases in nuclear PHB2 levels with Klf9 ablation, whereas at dpc $4 \cdot 5$, nuclear PHB2 expression for all cell types was independent of Klf9 (Fig. 5C).

\section{PHB2 expression in HESC with Klf9 knockdown}

To evaluate if KLF9 directly inhibits PHB2 expression in an $\mathrm{E}_{2}$-dominated environment as suggested in vivo (above), a HESC was used in siRNA targeting of human KLF9 mRNA. PHB2 expression in siKlf9 mRNA-transfected cells, relative to those transfected with non-specific (scrambled) siRNAs, was subsequently determined at the transcript and nuclear protein levels. Transfection of HESC with a pool of Klf9 siRNAs (Dharmacon) followed by $\mathrm{E}_{2}$ treatment diminished Klf9 gene expression by at least $70 \%$, relative to similarly treated cells transfected with scrambled siRNAs (Fig. 6A); this level of knockdown at the transcript level resulted in a comparable decrease in KLF9 protein levels, as determined by western blots (Fig. 6B). The decrease in Klf9 expression significantly $(P=0 \cdot 009)$, albeit modestly increased (by 20\%) PHB2 transcript levels (Fig. 6A). Immunofluorescence studies with anti-PHB2 antibody confirmed the up-regulated expression of PHB2 at the level of the protein (Fig. 6C). Relative to scrambled siRNA, Klf9 siRNA increased the percentage of nuclear-localized PHB2 in $\mathrm{E}_{2}$-treated HESC (Fig. 6D). 

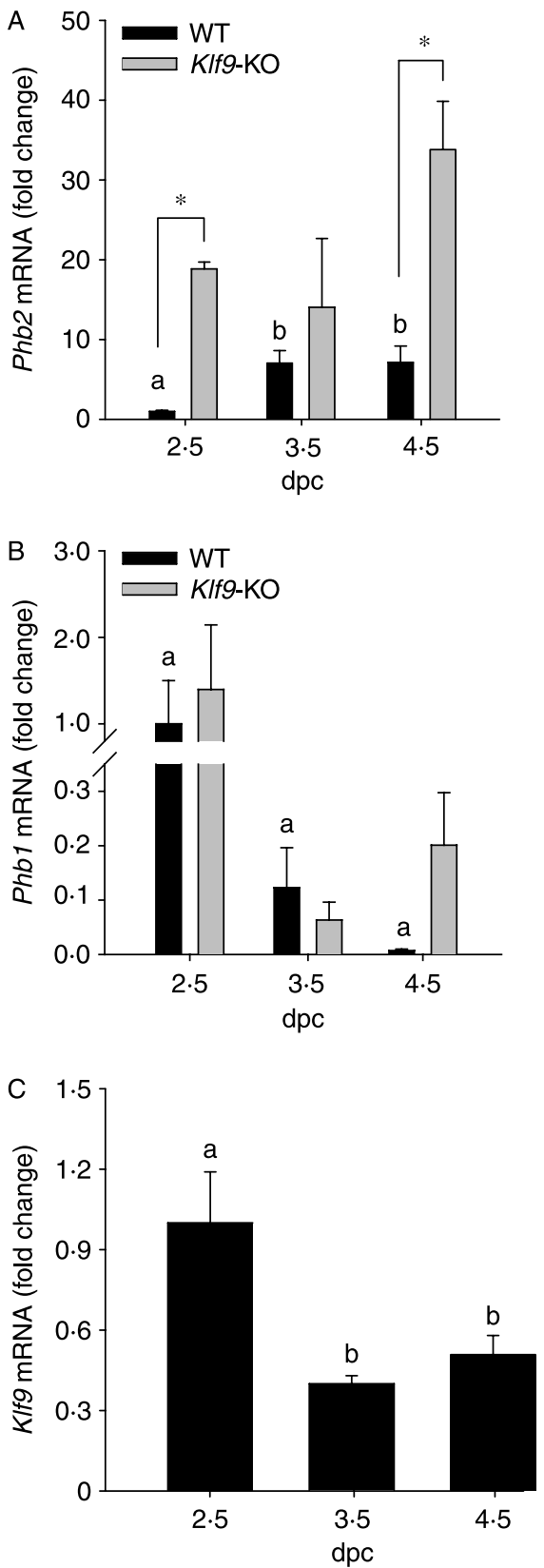

Figure 4 Uterine transcript levels for (A) Phb2 and (B) Phb1 in WT and Klf9 null mice and for (C) KIf9 in WT mice during early pregnancy. mRNA expression was quantified by qPCR and normalized to that of the control gene Ppia. Transcript levels (mean \pm S.E.M.) were determined at dpc 2.5, 3.5, and 4.5 in WT (Phb2, Phb1, and Klf9) and Klf9 KO (Phb2 and Phb1) mice and expressed as fold change relative to WT (dpc $2 \cdot 5)$ group. Significant difference $\left(^{*}\right)$ due to genotype at $P<0 \cdot 05$ was identified by Student's $t$-test for dpc $2 \cdot 5$ and $4 \cdot 5$. One-way ANOVA was used to compare Phb2 and Phb1 mRNA levels of WT among pregnancy days. Means with different superscripts differed at $P<0 \cdot 05$.
Parallel studies using anti-ESR 1 antibody showed no effect of KLF9 status on the nuclear levels of this protein (data not shown), consistent with the findings for uterine ST cells of Ovx, $\mathrm{E}_{2}$-treated Klf9 null mice (described in text, above).

\section{Discussion}

Results from the present study suggest the participation of the transcription factor KLF9 in paracrine signaling to regulate $\mathrm{E}_{2}$-induced proliferation of uterine endometrial epithelial cells. Using Ovx, $\mathrm{E}_{2}$-treated Klf9 null mutant mice and corresponding WT littermates as controls, we showed that the ST KLF9 regulation of the proliferative response of endometrial LE cells to $\mathrm{E}_{2}$ is negatively associated with uterine Phb2 expression and nuclear localization of PHB2 in endometrial epithelial cells. We confirmed the physiological relevance of the negative linkage between KLF9 and PHB2 by demonstrating that the transiently lower proliferative response of uterine endometrial epithelial cells of Klf9 null mutants relative to WT counterparts at dpc $2 \cdot 5$ (Velarde et al. 2005) corresponded with increased expression of nuclear PHB2 expression in these cells. Finally, we demonstrated that in the $E_{2}$-treated HESC, a reduction in KLF9 expression resulted in increased transcript and nuclear protein levels for PHB2. Taken together, our results suggest a working model whereby $E_{2}$ control of epithelial cell proliferation can be dynamically influenced by ST KLF9 (Fig. 7). By attenuating expression of ST ESR 1 corepressor PHB2 that can interact with ESR1 coactivators such as NCOA1 (Montano et al. 1999) and histone deacetylases (Kurtev et al. 2004), KLF9 may promote ESR1-mediated transactivation of yet unknown paracrine factor(s) to repress epithelial expression of PHB2, leading to increased proliferative responsiveness to $\mathrm{E}_{2}$.

The present study provides the first direct evidence for KLF9 involvement in $E_{2}$-induced proliferation of uterine endometrial cells. Our previous studies using early pregnant mouse uteri hinted at this possibility, since maximal BrdU labeling of endometrial epithelial cells occurred at the E-dominated uterine environment of dpc $2 \cdot 5$ (Velarde et al. 2005), coincident with the highest uterine ST Klf9 expression (this study). Since uterine expression of Klf9 is predominantly ST and is lacking in luminal epithelial cells (Simmen et al. 2004), the absence of proliferative response to $E_{2}$ of the latter endometrial compartment in Ovx, $\mathrm{E}_{2}$-treated Klf9 null mice suggests paracrine control by an $\mathrm{E}_{2}$-induced $\mathrm{ST}$-derived growth-regulatory factor(s) whose synthesis may involve functional KLF9/ESR1 interactions. The latter is in agreement with previous reports that ST-localized ESR1 mediates the mitogenic effects of $\mathrm{E}_{2}$ on neighboring LE cells (Cooke et al. 1997) and that KLF9 can influence ESR1 transactivity in endometrial epithelial cells (Velarde et al. 2007). Although the identity of this putative KLF9/ESR1-regulated paracrine factor(s) is currently unknown, our results suggest that this factor(s) may be involved in the inhibition of PHB2 expression in luminal epithelial cells to allow these cells, which express 
A

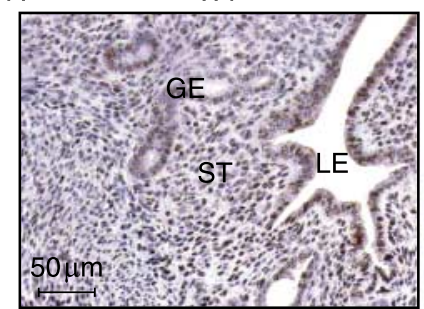

B

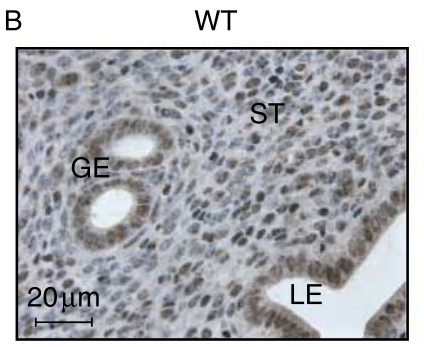

C

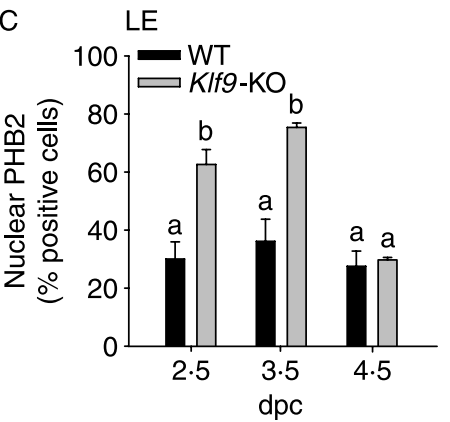

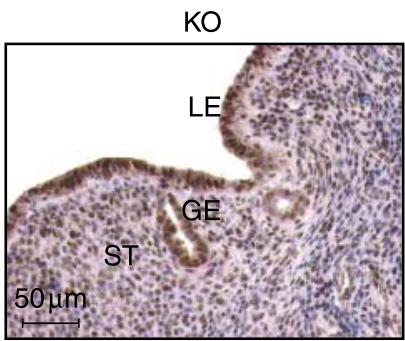
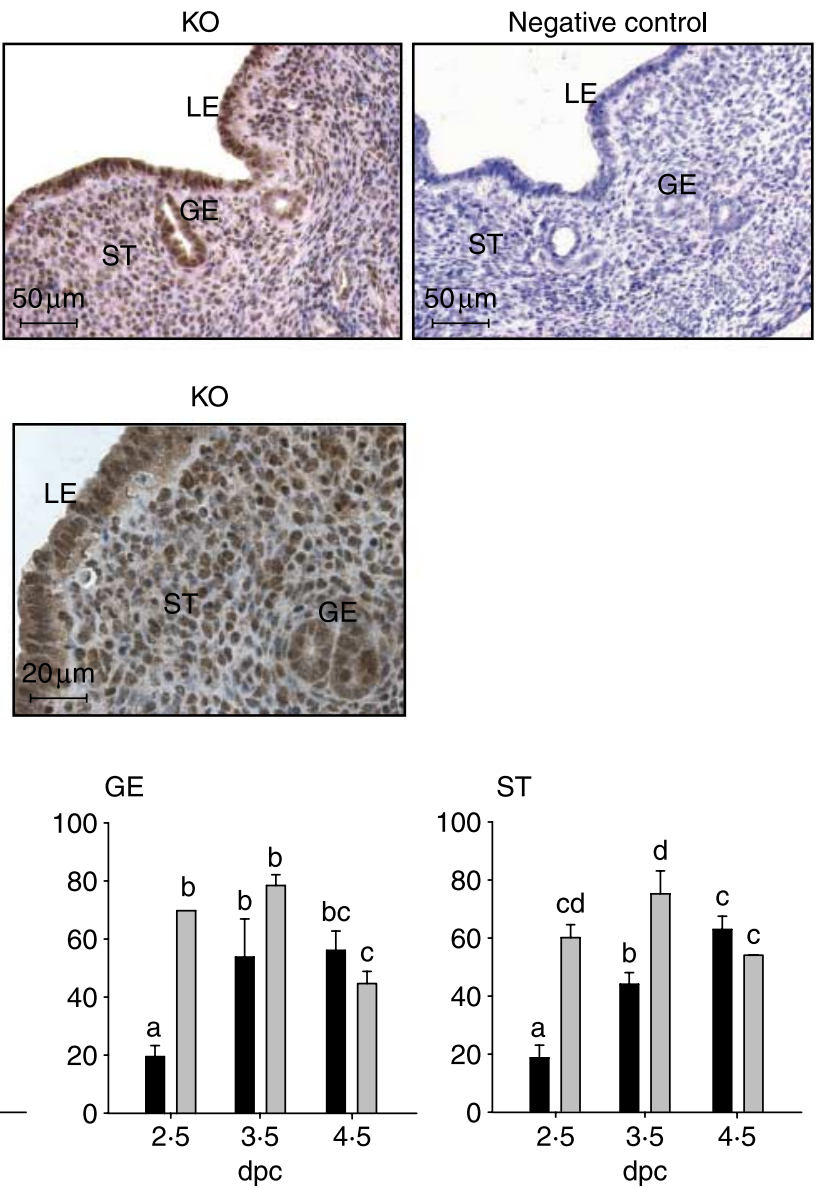

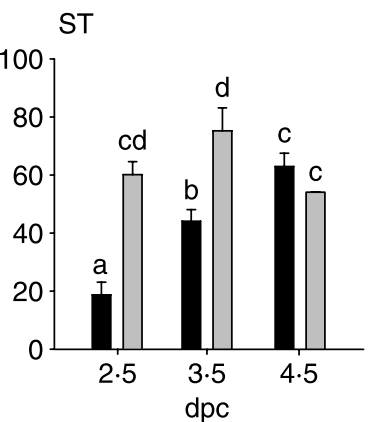

Figure 5 Nuclear PHB2 levels in uterine endometrial cells of WT and Klf9 null mice during early pregnancy. (A) Representative immunostaining for uterine PHB2 in pregnant WT and KIf9 null mice at dpc 2.5. GE, glandular epithelium; LE, luminal epithelium; ST, stroma. Panels are shown at $200 \times$ magnification. Negative control was tissue from Klf9 KO mice processed similarly except for omission of primary antibody. (B) Representative PHB2 immunostaining of GE, LE, and ST cells of pregnant WT and Klf9 null (KO) mice at dpc 2.5 are shown at $400 \times$ magnification to demonstrate nuclear localization of immunoreactive PHB2. (C) The percentages of nuclear immunostained cells are presented as mean \pm S.E.M. ( $n=3-5$ mice per treatment per genotype). Significant differences were identified by two-way ANOVA, followed by Tukey's test. Means with different superscripts differed at $P<0 \cdot 05$.

ESR1, to optimally respond to $\mathrm{E}_{2}$. Interestingly, the negative relationship between PHB2 and KLF9 was not extended to the related Phb1 gene, whose gene product also functions as a repressor of ESR 1 signaling (Mishra et al. 2006, He et al. 2007). However, we cannot exclude the possibility that an association between KLF9 and PHB1 expression may be manifest at the level of PHB1 protein.

To further investigate the molecular interplay between ESR 1 and KLF9 in ST cells, we evaluated the effect of Klf9 knockdown as a function of $\mathrm{E}_{2}$ treatment on $\mathrm{PHB} 2$ expression using the HESC. Since loss of KLF9 expression in vivo was associated with enhanced expression of nuclear PHB2 in ST (by 40-fold), more so than in GE and LE (by threefold) cells, it is possible that KLF9 regulation of PHB2 levels in ST may be distinct from that in LE. Our findings that abrogation of Klf9 gene expression resulted in increased PHB2 transcript and nuclear PHB2 protein levels confirm the negative association of KLF9 and PHB2 in vivo. However, the in vitro effects were modest and did not recapitulate the two- to threefold (pregnancy) and 40-fold (Ovx/ $\mathrm{E}_{2}$ model) increases in PHB2 expression seen in vivo. Albeit this difference may be a function of the limited time of exposure to $\mathrm{E}_{2}$ in vitro, the in vivo data were also observed $24 \mathrm{~h}$ post- $\mathrm{E}_{2}$, raising the strong possibility of a requirement for ST and LE communication (paracrine signaling) in this $\mathrm{E}_{2}$-mediated process.

Our studies indicate that the regulation of uterine PHB2 expression involving KLF9 is complex and could occur at the levels of RNA, protein, and cellular localization. Indeed, the lack of a clear-cut association between nuclear PHB2 and KLF9 expression (this study) with LE proliferation status 
A
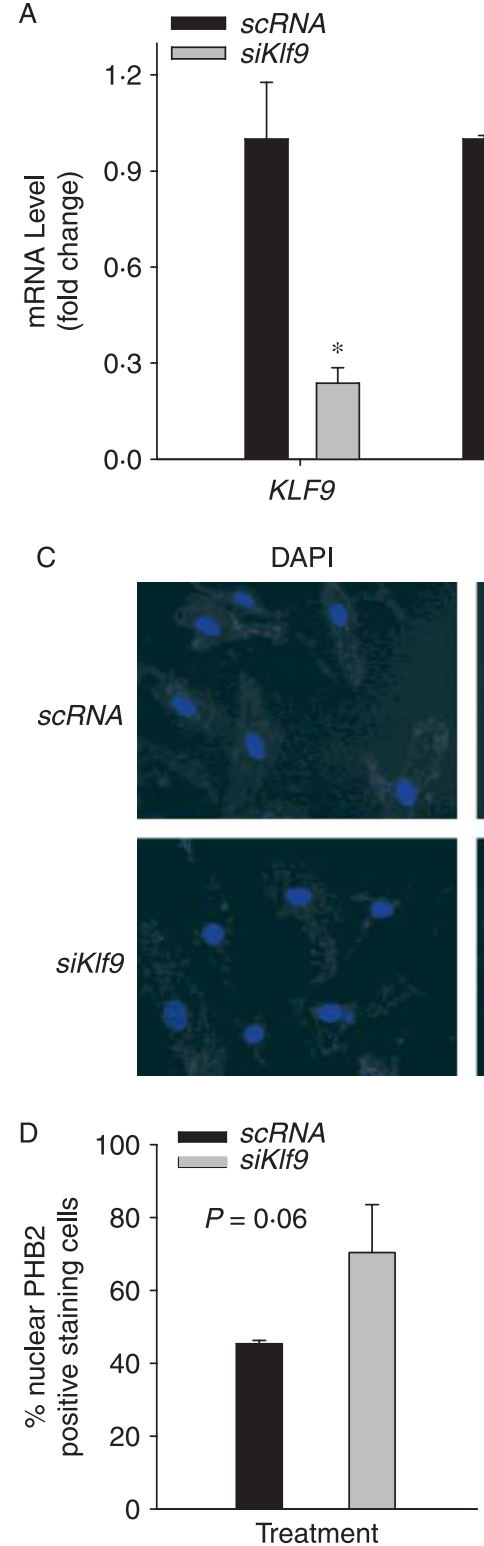

B

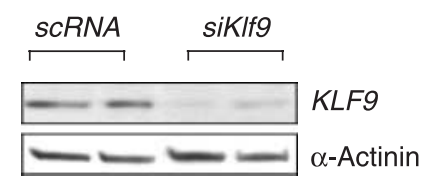

PHB2
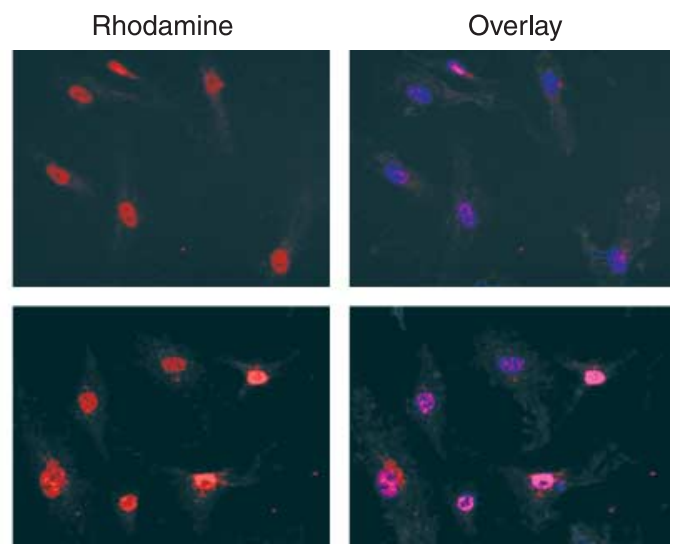

Figure 6 PHB2 levels in human endometrial stromal cells as a function of KLF9 expression status. HESC were incubated in phenol-red free DMEM/Ham's F12 medium containing 10\% charcoal-stripped serum and $E_{2}(10 \mathrm{nM})$ in the presence of siRNA to scrambled (negative control) mRNA or siRNA to KLF9 $(50 \mathrm{nM})$. (A) Harvested cells were analyzed for KLF9 and PHB2 transcripts by qPCR and normalized to control gene Rpl7. Results (mean \pm s.E.M.; relative to cells treated with scrambled siRNA) shown are representative of two to three independent experiments, with each experiment conducted in triplicates. (B) Western blots of whole cell lysates prepared from HESC cells as a function of Klf9 knockdown. Cells transfected with siRNA to scrambled sequence (negative control) or to Klf9 were subjected to western blots using anti-KLF9 or anti- $\alpha$-actinin antibodies. Each lane represents an independent experiment. (C) HESC treated with $\mathrm{E}_{2}$ for $24 \mathrm{~h}$ were immunostained for PHB2/REA and counterstained for DAPI. Immunopositive cells were visualized using fluorescent antibodies. Overlay of anti-PHB2 (red) and DAPI (blue) staining cells showed nuclear localization of PHB2. (D) The percentages of nuclear PHB2 staining cells were expressed relative to the total number of cells counted. Results (mean \pm s.E.M.) were normalized to those of scrambled siRNA-treated cells and were from two independent experiments, with each experiment performed in triplicates. Differences between groups were determined by $t$-test $(P=0 \cdot 06)$. 


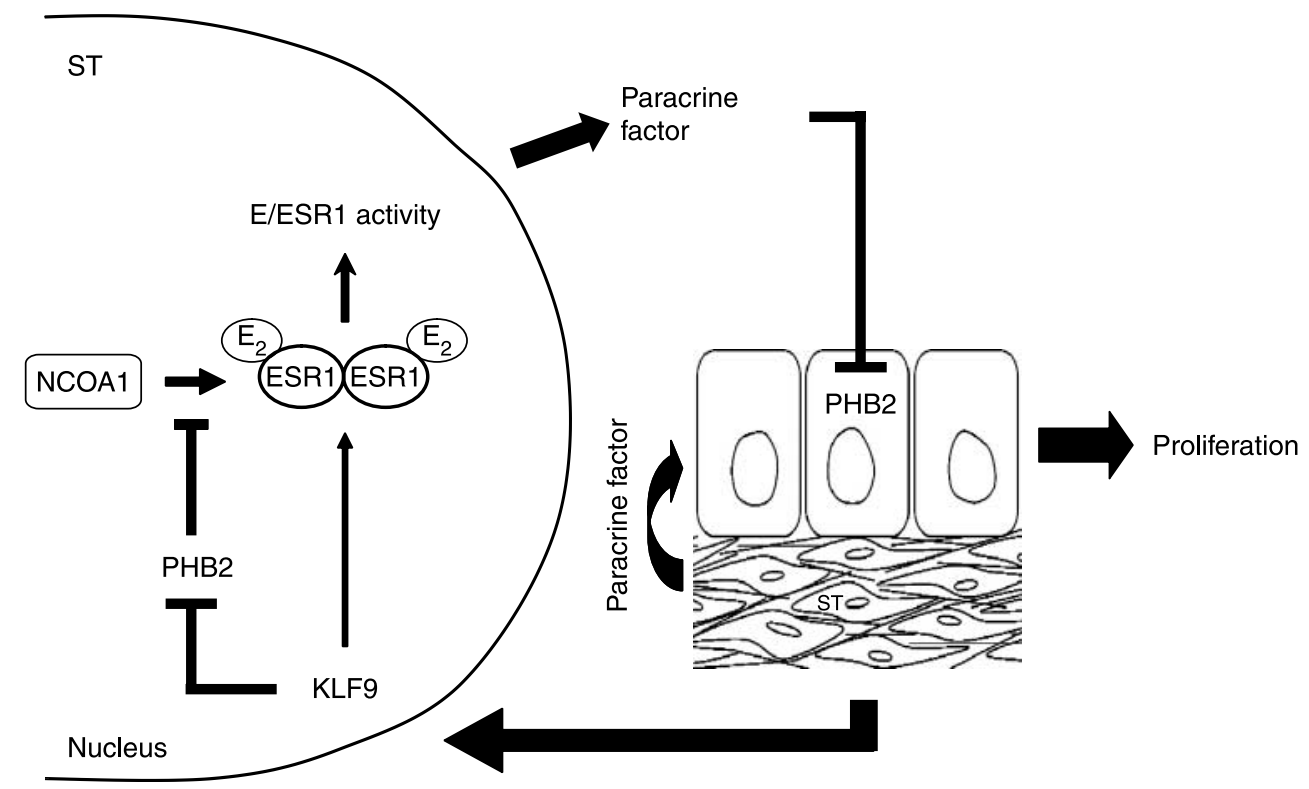

Figure 7 Postulated model for $\mathrm{E}_{2}$ control of uterine epithelial cell proliferation involving stromal KLF9. By attenuating expression of stromal corepressor PHB2, which can inhibit the interaction of ESR1 coactivators such as NCOA1 with ESR1, KLF9, may promote ligand-dependent ESR1-mediated transactivation of paracrine factor(s) yet unknown to repress epithelial expression of PHB2, leading to cell proliferation.

(Velarde et al. 2005) at dpc $3 \cdot 5$ and $4 \cdot 5$, in contrast to that seen at $\mathrm{dpc} 2 \cdot 5$, and the loss of the inverse association of KLF9 and nuclear PHB2 levels at dpc 4.5 in LE as well as in GE and ST, suggests the additional contributions of progesterone, other KLF9-related proteins that may compensate for the loss of KLF9 function (Simmen et al. 2004) and possibly, the attaching embryo in the control of LE proliferation at early pregnancy. Since control of PHB2 levels may constitute part of a homeostatic mechanism to allow for the appropriate proliferative response of uterine cells to $E_{2}$, further investigations into the function and molecular regulation of PHB2 in the uterine endometrium by KLF9 in concert with other nuclear steroid receptor co-regulators should constitute an important area for future research.

In summary, our studies define a potential mechanism whereby control of E-induced endometrial epithelial cell proliferation can be dynamically regulated by ST KLF9 through its indirect inhibition of nuclear PHB2 expression in epithelial cells. Results suggest the participation of KLF9 in $\mathrm{E}_{2}$-dependent synthesis of an ST-derived paracrine factor(s) that mediates this epithelial mitogenic response to estrogen. While the global significance of KLF9 regulation of ESR1 signaling mediated by PHB2 in luminal epithelial cells is currently unclear, the sub-fertility phenotype of Klf9 null mice (Simmen et al. 2004) suggests physiological relevance of KLF9 during the $\mathrm{E}_{2}$-dominated period of early pregnancy prior to implantation (i.e., predecidual ST) and underscores the delicate control of nuclear coactivator and corepressor expression to achieve the requisite uterine response to estrogen for successful pregnancy.

\section{Declaration of interest}

The authors declare that there is no conflict of interest that could be perceived as prejudicing the impartiality of the research reported.

\section{Funding}

This work was supported by the National Institutes of Health grant no. HD21961.

\section{References}

Cooke PS, Buchanan DL, Young P, Setiawan T, Brody J, Korach KS, Taylor J, Lubahn DB \& Cunha GR 1997 Stromal estrogen receptors mediate mitogenic effects of estradiol on uterine epithelium. PNAS 94 6535-6540. Delage-Morroux R, Martini PG, Choi I, Kraichely DM, Hoeksema J \& Katzenellenbogen BS 2000 Analysis of estrogen receptor interaction with repressor of estrogen receptor activity (REA) and the regulation of estrogen receptor transcriptional activity by REA. Journal of Biological Chemistry $\mathbf{2 7 5}$ 35848-35856.

Dupont S, Krust A, Gansmuller A, Dierich A, Chambon P \& Mark M 2000 Effect of single and compound knockouts of estrogen receptors alpha (ER $\alpha)$ and beta $(E R \beta)$ on mouse reproductive phenotypes. Development 127 4277-4291.

Dutertre M \& Smith CL 2003 Ligand-independent interactions of $\mathrm{p} 160 /$ steroid receptor coactivators and CREB-binding protein (CBP) with estrogen receptor $\alpha$ : regulation by phosphorylation sites in the A/B region depends on other receptor domains. Molecular Endocrinology 17 1296-1314.

Font de Mora J \& Brown M 2000 AIB1 is a conduit for kinase-mediated growth factor signaling to the estrogen receptor. Molecular and Cellular Biology 20 5041-5047.

Fusaro G, Dasgupta P, Rastogi S, Joshi B \& Chellappan S 2003 Prohibitin induces the transcriptional activity of $\mathrm{p} 53$ and is exported from the nucleus upon apoptotic signaling. Journal of Biological Chemistry 278 47853-47861. 
Gamble SC, Chotal D, Odontiadis M, Dart DA, Brooks GN, Powell SM, Reebye V, Varela-Carver A, Kawano Y, Waxman J et al. 2007 Prohibitin, a protein down-regulated by androgens, represses androgen receptor activity. Oncogene 26 1757-1768.

Green KA \& Carroll JS 2007 Oestrogen-receptor-mediated transcription and the influence of co-factors and chromatin state. Nature Reviews. Cancer $\mathbf{7}$ 713-722.

Hall JM, Couse JF \& Korach KS 2001 The multifaceted mechanisms of estradiol and estrogen receptor signaling. Journal of Biological Chemistry 276 36869-36872.

He B, Feng Q, Mukherjee A, Lonard DM, DeMayo FJ, Katzenellenbogen BS, Lydon JP \& O'Malley BW 2007 A repressive role for prohibitin in estrogen signaling. Molecular Endocrinology 22 344-360.

Hu YC, Shyr CR, Che W, Mu XM, Kim E \& Chang C 2002 Suppression of estrogen receptor-mediated transcription and cell growth by interaction with TR2 orphan receptor. Journal of Biological Chemistry 277 33571-33579.

Imataka H, Sogawa K, Yasumoto K, Kikuchi K, Sasano K, Kobayashi A, Hayami M \& Fujii-Kuriyama Y 1992 Two regulatory proteins that bind to the basic transcription element (BTE). A GC box sequence in the promoter region of the rat P4501A1 gene. EMBO Journal 11 3663-3671.

Krikun G, Mor G, Alvero A, Guller S, Schatz F, Sapi E, Rahman M, Caze R, Qumsiyeh M \& Lockwood CJ 2004 A novel immortalized human endometrial stromal cell line with normal progestational response. Endocrinology 145 2291-2296.

Kurtev V, Margueron R, Kroboth K, Ogris E, Cavailles V \& Seiser C 2004 Transcriptional regulation by the repressor of estrogen receptor activity via recruitment of histone deacetylases. Journal of Biological Chemistry 279 24384-24843

Lonard DM \& O'Malley BW 2006 The expanding cosmos of nuclear receptor coactivators. Cell 125 411-414.

McKenna NJ \& O'Malley BW 2002 Combinatorial control of gene expression by nuclear receptors and coregulators. Cell 108 465-474.

Mishra S, Murphy LC \& Murphy LJ 2006 The prohibitins: emerging roles in diverse functions. Journal of Cellular and Molecular Medicine 10 353-363.

Montano MM, Ekena K, Delage-Morroux R, Chang W, Martini P \& Katzenellenbogen BS 1999 An estrogen receptor-selective coregulator that potentiates the effectiveness of antiestrogens and represses the activity of estrogens. PNAS 96 6947-6952.

Morita M, Kobayashi A, Yamashita T, Shimanuki T, Nakajima O, Takahasi S, Ikegami S, Inokuchi K, Yamashita K, Yamamoto M et al. 2003 Functional analysis of basic transcription element binding protein by gene targeting technology. Molecular and Cellular Biology 23 2489-2500.

Mussi P, Liao L, Park S-E, Ciana P, Maggi A, Katzenellenbogen BS, Xu J \& O'Malley BW 2006 Haploinsufficiency of the corepressor of estrogen receptor activity (REA) enhances estrogen receptor function in the mammary gland. PNAS 103 16716-16721.

Park SE, Xu J, Frolova A, Liao L, O'Malley BW \& Katzenellenbogen BS 2005 Genetic deletion of the repressor of estrogen receptor activity (REA) enhances the response to estrogen in target tissues in vivo. Molecular and Cellular Biology 25 1989-1999.

Simmen RCM, Eason RR, McQuown JR, Linz AL, Kang T-J, Chatman L, Till SR, Fujii-Kuriyama Y, Simmen FA \& Oh SP 2004 Subfertility, uterine hypoplasia, and partial progesterone resistance in mice lacking the Krüppellike factor $9 /$ basic transcription element-binding protein-1 (Bteb1) gene. Journal of Biological Chemistry 279 29286-29294.
Stenoien DL, Nye AC, Mancini MG, Patel K, Dutertre M, O'Malley BW, Smith CL, Belmont AS \& Mancini A 2001 Ligand-mediated assembly and real-time cellular dynamics of estrogen receptor $\alpha$-coactivator complexes in living cells. Molecular and Cellular Biology 21 4404-4412.

Suske G, Bruford E \& Philipsen S 2005 Mammalian SP/KLF transcription factors: bring in the family. Genomics 85 551-556.

Tsai MJ \& O'Malley BW 1994 Molecular mechanisms of action of steroid/thyroid receptor superfamily members. Annual Review of Biochemistry 63 451-486.

Velarde MC, Geng Y, Eason RR, Simmen FA \& Simmen RCM 2005 Null mutation of Krüppel-like factor $9 /$ basic transcription element binding protein-1 alters peri-implantation uterine development in mice. Biology of Reproduction 73 472-481.

Velarde MC, Iruthayanathan M, Eason RR, Zhang D, Simmen FA \& Simmen RCM 2006 Progesterone receptor transactivation of the secretory leukocyte protease inhibitor gene in Ishikawa endometrial epithelial cells involves recruitment of Krüppel-like factor 9/Basic transcription element binding protein-1. Endocrinology 147 1969-1978.

Velarde MC, Zeng Z, McQuown JR, Simmen FA \& Simmen RCM 2007 Krüppel-like factor 9 is a negative regulator of ligand-dependent estrogen receptor $\alpha$ signaling in Ishikawa endometrial adenocarcinoma cells. Molecular Endocrinology 21 2988-3001.

Wang S, Zhang B \& Faller DV 2004 BRG1/BRM and prohibitin are required for growth suppression by estrogen antagonists. EMBO Journal $\mathbf{2 3}$ 2293-2303.

Wang S, Zhang C, Nordeen SK \& Shapiro DJ 2007 In vitro fluorescence anisotropy analysis of the interaction of full-length SRC1a with estrogen receptors $\alpha$ and $\beta$ support an active displacement model for coregulator utilization. Journal of Biological Chemistry 282 2765-2775.

Yahata T, Shao W, Endoh H, Hur J, Coser KR, Sun H, Ueda Y, Kato S, Isselbacher KJ, Brown M et al. 2001 Selective coactivation of estrogendependent transcription by CITED1 CBP/p300-binding protein. Genes and Development 15 2598-2612.

Zhang D, Zhang X-L, Michel FJ, Blum JL, Simmen FA \& Simmen RCM 2002 Direct interaction of the Krüppel-like family (KLF) member, BTEB1 and progesterone receptor mediates progesterone-responsive gene expression in endometrial epithelial cells. Endocrinology 141 62-73.

Zhang X-L, Zhang D, Michel FJ, Blum JL, Simmen FA \& Simmen RCM 2003 Selective interactions of KLF9/BTEB1 with progesterone receptor isoforms A and B determine transcriptional activity of progesteroneresponsive genes in endometrial epithelial cells. Journal of Biological Chemistry 278 2174-21482.

Zheng FF, Wu RC, Smith CL \& O'Malley BW 2005 Rapid estrogen-induced phosphorylation of the SRC-3 coactivator occurs in an extranuclear complex containing estrogen receptor. Molecular and Cellular Biology 25 $8273-8284$

Received in final form 28 September 2008

Accepted 1 October 2008

Made available online as an Accepted Preprint

2 October 2008 